\title{
Integration of test procedures and trials for DECT handsets
}

\author{
A. Alonistioti, P. Kostarakis, K. Dangakis, A. A. Alexandridis, \\ A.Paschalis, N. Gaitanis, S. Xiroutsikos, E. Adilinis, \\ A. Vlahakis, P. Katrivanos \\ N.C.S.R. "Demokritos", Institute of Informatics and \\ Telecommunications, 15310 Ag. Paraskevi, Athens, Greece. \\ TEL: +3016520847, FAX: +3016532175 \\ e-mail: kdang@iit.nrcps.ariadne-t.gr
}

\begin{abstract}
In this paper we elaborate on a generic integrated reference modelling of trials execution procedure and test procedures for the Digital European Cordless Telecommunications (DECT) physical layer conformance testing. This model provides for a generic reference approach to trials execution steps and test case selection, based on the specific physical entities that have to be tested. Trials are presented, in the form of elementary test procedures pertinent to a specific physical entity that has to be tested (i.e., time, frequency, power) and auxiliary test procedures for synchronisation, calibration, etc.
\end{abstract}

\section{Keywords}

DECT, Conformance Testing, Test Procedures, Integration, Modelling 


\section{INTRODUCTION}

For the deployment of the CTS-3/DECT test laboratories aiming to provide a conformance test service on DECT equipment in Europe, a test system and corresponding test suite have been developed. The framework for launching the DECT test facilities adheres to the ISO/IEC 9646 standard testing methodology (ISO/IEC 9646, 1993).

In this paper, the integration of trials execution procedure with respect to test procedures is introduced and a generic model for the mapping of elementary test procedures to trials execution steps is presented. This integration aims to advance the level of understanding of DECT test execution aspects in the communications industry and provide a framework addressed to formal/informal reference modelling development methodology for test procedures and trials execution mapping.

\section{TEST SYSTEM CONFIGURATION}

Conformance testing of the DECT physical layer involves the measuring of a physical entity (e.g. a modulated RF signal or a bit pattern) of the physical medium, that is, of the air interface. Thus, the DECT physical layer conformance testing requires an Equipment Under Test (EUT) and a Lower Test Unit (LTU) for controlling and observing the EUT through the air interface. All relevant procedures are described in (ISO/IEC 9646, 1993), (Alexandridis, 1995), (CEC/CTS3/DECT, 1994).

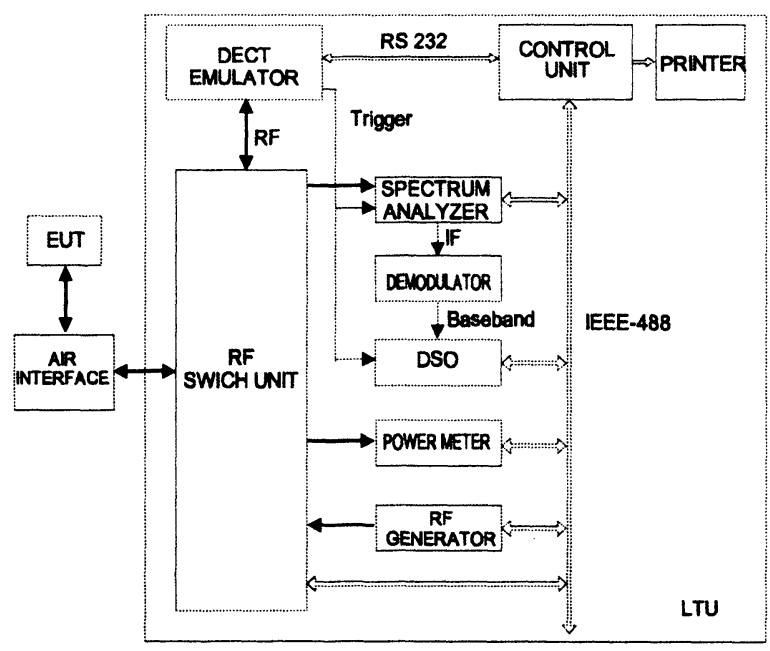

Figure 1 Test system configuration. 
The configuration of LTU, that we have developed for performing the DECT physical layer conformance test suite is shown in Figure 1 as a part of the whole test system, based on (Papavramidis, 1992), (CEC/CTS3/DECT 1992). The EUT in our case is a DECT handset.

\section{INTEGRATION OF TEST PROCEDURES INTO TRIALS}

The test case execution tool is the "heart" of the integrated DECT test system. The test case execution tool is implemented in the form of test procedures. Every test procedure is considered as a primitive software module related with the physical entity that has to be tested according to the specific test case. Such entities are time (e.g., measurements of jitter), frequency (e.g., measurements of accuracy of RF carriers) and power (e.g., measurements of spurious emissions). In that sense, the following three basic categories of test procedures are defined and described in (Alexandridis, 1995): time test procedures, frequency test procedures, power test procedures In addition to the elementary test procedures, there is also another category of auxiliary test procedures. They include software modules for the initialization and setting of the test system's instruments and units, the selection of the appropriate RF signal path through the RF switch unit, the control and programming of the DECT Emulator. Every executable test case consists of one or more of the elementary test procedures that are accompanied by other auxiliary test procedures. This way the test cases can form groups with respect to the category of the test procedures used in each test case. Each group is symbolised by the initial letters of the test procedures used for a complete test execution ( $T$ : time test procedures, F: frequency test procedures, $\mathrm{P}$ : power test procedures and A: auxiliary test procedures).The corresponding trials execution can be mapped on the respective group test procedures. Four groups can be identified. The 4 groups are characterised by the test procedures they comprise, namely, 1) the TPA group, 2) the TFA group, 3) the TA group and 4) the PA group.

All the DECT test cases currently covered by the test case execution tool in association with the 4 test groups previously defined are given below:

TPA : Transmitter Release/Attack Time, Transmitter Minimum/Maximum Power, Maintenance of Transmission after Packet End, Transmitter idle power output, Peak Power per Transceiver.

TFA : RF Carrier Modulation, Accuracy and Stability of RF Carriers.

TA : Timing Accuracy and Stability (Jitter from slot-to-slot), Reference Timer Accuracy and Stability of a RFP.

PA : Emissions due to Modulation, Emissions due to Transmitter Transients, Spurious Emissions when allocated a transmit channel.

These test procedures are based on those described abstractly in (prTBR6, 1992). The trials execution procedures for each group are illustrated in Figures 2 and 3. The main basic steps for complete test execution and the values returned by each step are also indicated. 

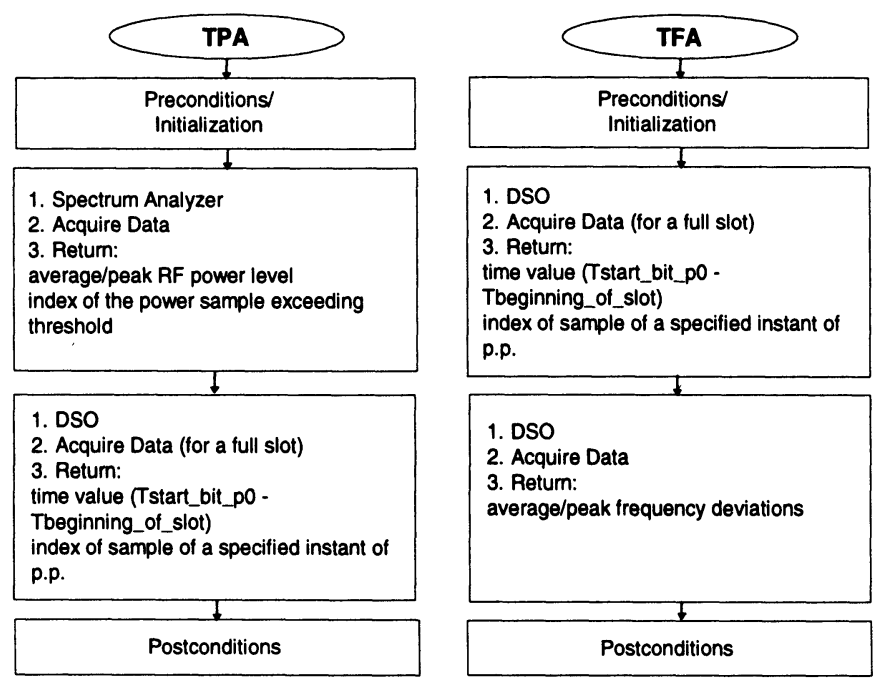

Figure 2 Trials execution procedures for the TFA and TPA groups.
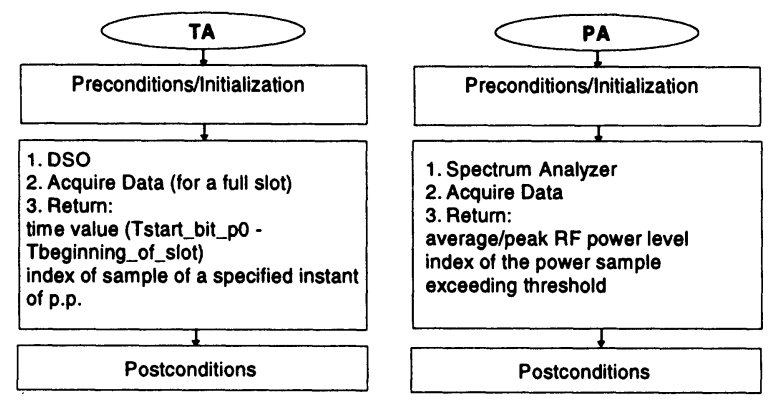

Figure 3 Trials execution procedures for the TA and PA groups.

Integration of the test procedures and trials provide an overview reference modelling of the manner in which trials are executed with respect to general, elementary test procedures. This aims at providing a common view with the communications industry of the test execution procedures to be followed during the DECT test service provisioning. Another target is to facilitate test selection with focus on the intended entities to be tested on the handset. The mapping of test procedures to trials execution procedures for the TPA and TFA test case groups are introduced in Figure 4. 

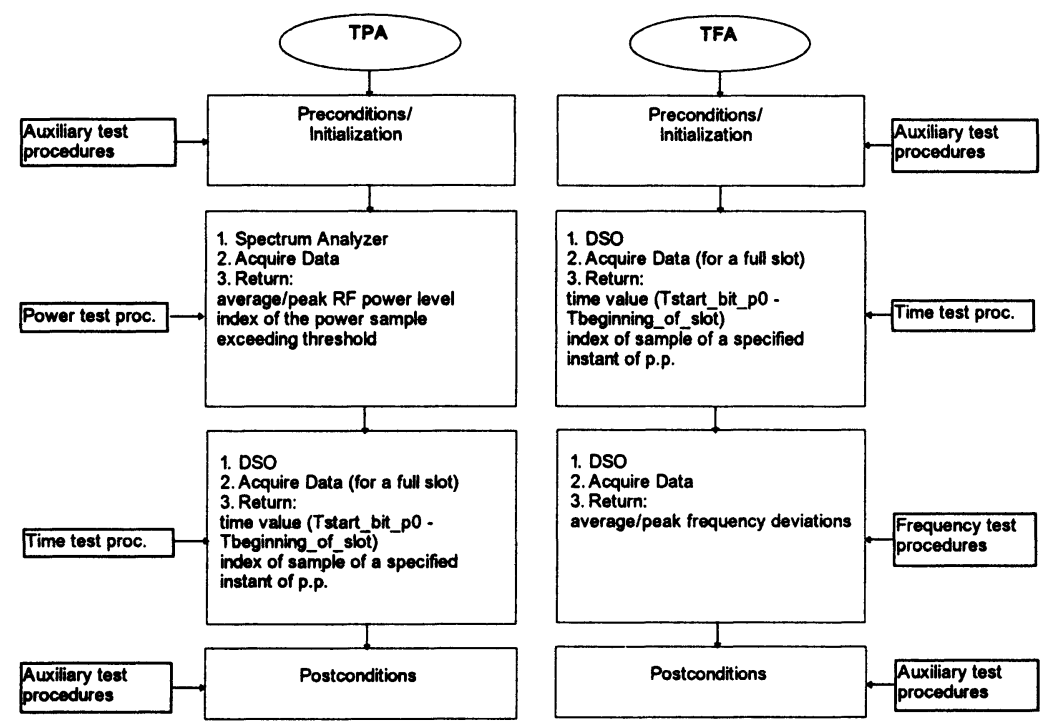

Figure 4 Trials execution procedures for the TFA and TPA groups and mapping of test procedures to trials execution procedures.

The mapping for the TA and PA test case groups is performed in a similar way. The mapping of test procedures and trials execution procedure provides a generic, integrated reference model for the trials execution steps with respect to test procedures and test case groups involved. The integrated model is illustrated in Figure 5. This provides for a generic evolutionary trials execution procedure and for efficient overview of test selection.

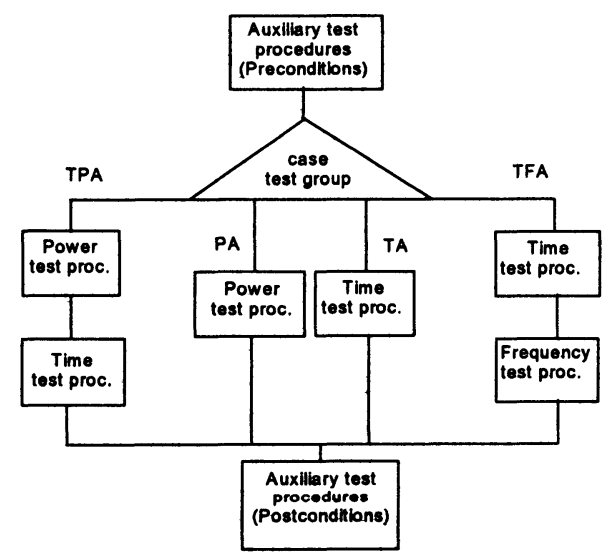

Figure 5 Integration of trials execution procedures and test procedures groups. 
Test suites, covering all the necessary test cases for DECT physical layer conformance testing, have been carried out on an equipment (DECT - handset) that was provided by a certain manufacturer. The use of the generic overview of trials execution procedures with respect to test procedures in the implementation of executable test cases is clarified below by describing, step-by-step, the execution of a specific test case.

The test case belongs to the TFA group and refers to measurement of accuracy and stability of RF carriers, that is measurement of the DECT portable part carrier frequency relative to an absolute frequency reference, set to the nominal frequency of the corresponding DECT RF channel. The steps during the execution of this test case are the following:

Using auxiliary test procedures, the DSO is initialised and programmed to start data acquisition at the beginning of a specific slot carrying the physical packet transmitted by the EUT. First, the frequency calibration module of the frequency test procedures generates the appropriate look-up table that maps the voltage levels at the demodulator output to frequency deviations from the nominal carrier frequency. The RF Switch Unit is programmed to select the appropriate RF signal path. The DECT Emulator (DECT Fixed Part) is given the command to establish a communication with the EUT (DECT Portable Part) in a specified channel and slot. As a result, the EUT is placed in a test mode whereby it performs the loopback function.

Using time test procedures, the DSO starts digitising the received physical packet for a full-slot duration and the acquired data are recorded. Then, the time, $\mathrm{T}_{0}$, of the start of bit $\mathrm{p} 0$ (first bit of the physical packet) is returned. This time reference is used to specify a time window containing only the bits of the loopback field of a packet transmitted by the EUT during the first $1 \mathrm{sec}$ after its transition from a non-transmitting mode to a transmit mode.

Using frequency test procedures the captured data contained in the predefined time window are processed and the EUT carrier frequency is calculated as the average of the measured frequencies corresponding to the voltage levels of the acquired samples.

Using auxiliary test procedures, the postconditions are set and this measurement is repeated following the same procedures as above but in this case data acquisition starts after allowing the EUT to be in an active locked state for more than $1 \mathrm{sec}$. This is accomplished by a time procedure delaying properly the start of the physical packet data acquisition.

For the specific EUT that was used, the measured carrier frequency deviations for the two cases mentioned above were equal to $8.985 \mathrm{kHz}$ and $7.122 \mathrm{kHz}$ respectively. Since these values were within $\pm 100 \mathrm{kHz}$ and $\pm 50 \mathrm{kHz}$ of the nominal DECT carrier frequency, that are the limits set by the standard (ISO/IEC $9646,1993)$ for the two cases respectively, it is concluded that the EUT passed the test. 


\section{CONCLUSIONS}

For acquiring a compact view of the DECT test service offered by the CTS3/DECT test service laboratory, the test system configuration and the trials execution procedures were referenced. The elementary test procedures for DECT test cases execution were identified and an integrated reference model for the trials execution procedures modelling with respect to elementary test procedures was introduced in this paper. The model is evolutionary in the sense that it can be extended to cover also other test cases' execution that would cover future user needs. It is also generic, as it maps the test execution steps to elementary test procedures, providing a uniform way of execution. Furthermore, due to the methodology followed for its construction it provides an overview tool for test case selection. This model increases readability and understanding of test case execution procedures and serves as a base for common ground of understanding between the test service laboratory and the industrial customer for the trials provision.

\section{REFERENCES}

Alexandridis, A.A., Paschalis, A., Dangakis, K., Kostarakis, P., Lazarakis, F. and Gaitanis, N. (1995) Test Procedures for DECT Physical Layer Conformance Testing. International Conference on Telecommunications, ITC'95, Bali, Indonesia, 106-109.

CEC/CTS3/DECT project Deliverable 4 (1992) List of Equipment and Facilities. CEC/CTS3/DECT project Deliverable 11 (1994) Demonstration of Services.

ISO/IEC 9646 (1993) IT - OSI - Conformance Testing Methodology and Framework.

Papavramidis, A., Paschalis, A., Myrtue, O., Dangakis, K., Tombras, G. and Kostarakis, P. (1992) A Conformance Test System for DECT Physical Layer. Proc. of the $3^{\text {rd }}$ IEEE International Symposium on Personal, Indoor and Mobile Radio Communications, Boston, Massachusetts, 357-361.

\section{BIOGRAPHY}

Athanassia A. ALONISTIOTI is research assistant at the I.I.T. of the N.C.S.R. "DEMOKRITOS". Her current interests include third generation mobile communications (UMTS), SDL, formal methods and testing, modelling, simulation and design of object oriented platforms and protocols for advanced communications systems.

Panos D. KOSTARAKIS is research Director at the I.I.T. of the N.C.S.R. "DEMOKRITOS". He is actively involved in testing and type approval and he is Lloyd's registered auditor for ISO 9000 and member of the Total Quality forum. 
Kostas P. DANGAKIS is researcher at the I.I.T. of the N.C.S.R. "DEMOKRITOS", and now head of the Mobile Communications Laboratory. His current interests include Mobile Communications and, specifically, Digital modulation and data transmission techniques, Spread Spectrum Systems and CDMA techniques.

Antonis A. ALEXANDRIDIS is researcher at the I.I.T. of the N.C.S.R. "DEMOKRITOS", in Mobile Communications Lab. His current interests include Mobile Communications, Propagation Models, Digital Modulation Techniques, and, specifically, Spread Spectrum Systems and CDMA techniques.

Antonis M. PASCHALIS is researcher at the I.I.T. of the N.C.S.R. "DEMOKRITOS". His current interests include research in the area of Testing, Design for Testability, Built-In Self Test, Automatic Test Generation, SelfChecking and Conformance Testing.

Nikos GAITANIS is R\&D scientist at the I.I.T. of the N.C.S.R. "DEMOKRITOS", in the Hardware Technology Lab. His current interests include Conformance Testing, Formal Methods and Test case derivation.

Spyros K. XIROUTSIKOS is R\&D scientist at the I.I.T. of the N.C.S.R. "DEMOKRITOS", in the Hardware Technology Lab. His current interests include Design and Development of control and communication systems.

Eleftherios S. ADILINIS is a technician at the I.I.T. of the N.C.S.R. "DEMOKRITOS", in Mobile Communications Lab. His current interests include Design and Development of advanced electronic circuits and software.

Antonis B. Vlahakis is R\&D scientist at I.I.T. of the N.C.S.R. "DEMOKRITOS", in Mobile Communications Lab. His current interests are in the area of Communications, Power Electronics, Biomedical Electronics.

Panagotis L. Katrivanos is R\&D scientist at I.I.T. of the N.C.S.R. "DEMOKRITOS", in Mobile Communications Lab . His current interests are in the area of Mobile Communications, Propagation aspects, channel characterization, simulation and modelling. 